\title{
Dynamical Entropy Production in Spiking Neuron Networks in the Balanced State
}

\author{
Michael Monteforte* and Fred Wolf \\ Max Planck Institute for Dynamics and Self-Organization, Göttingen, Germany, \\ Faculty of Physics, Georg-August-University Göttingen, Göttingen, Germany, \\ Bernstein Center for Computational Neuroscience, Göttingen, Germany
}

\begin{abstract}
We demonstrate deterministic extensive chaos in the dynamics of large sparse networks of theta neurons in the balanced state. The analysis is based on numerically exact calculations of the full spectrum of Lyapunov exponents, the entropy production rate and the attractor dimension. Extensive chaos is found in inhibitory networks and becomes more intense when an excitatory population is included. We find a strikingly high rate of entropy production that would limit information representation in cortical spike patterns to the immediate stimulus response.
\end{abstract}

Neurons in the cerebral cortex fire action potentials (spikes) in highly irregular, seemingly random sequences [1]. Since neurons in isolation reliably respond to the repeated injection of identical temporally varying inputs [2], the irregular activity in the cortex is not believed to result from a randomness in the spike generating mechanism, but rather from strongly fluctuating synaptic inputs [3]. Several explanations for the origin of such fluctuating inputs have been proposed [4, 5]. The prevailing explanation is a dynamic balance between excitatory and inhibitory inputs, also known as the balanced state of cortical networks. Such a balance in neuronal circuits has been demonstrated experimentally in vitro and in vivo [6]. Its statistical characteristics have been studied theoretically in networks of excitatory and inhibitory neurons [5, 7] and in networks of only inhibitory neurons, where the recurrent inhibition balances external excitatory currents [8]. These studies established that in sparsely connected networks with relatively strong synapses the balanced state emerges robustly from the collective dynamics of the network, without an external source of randomness.

The dynamical nature of the balanced state, however, remains controversial and poorly understood. In a seminal paper, van Vreeswijk and Sompolinsky developed a powerful mean field theory of the balanced state for excitatoryinhibitory networks of binary neurons [5]. In their framework, nearby trajectories diverged faster than exponentially, demonstrating an extremely intense chaos with an infinite largest Lyapunov exponent. More recently, studies of inhibitory networks of leaky integrate and fire neurons reported stable chaos [8]. Stable chaos, originally discovered in coupled map lattices is characterized by irregular but non-chaotic dynamics with a negative definite Lyapunov spectrum [9].

Answering the question of whether the balanced state is chaotic is of fundamental importance for understanding information representation and processing in the cortex. If cortical networks were to generate stable complex firing patterns, these might serve as a coding space for storage and processing of, e.g., sensory information. If, alternatively, cortical networks operate in a chaotic regime, information processing would be intrinsically limited by the dynamical entropy production that turns microscopic perturbations such as ion channel noise into global firing patterns.
In this Letter, we present a comprehensive characterization of the balanced state in networks of $N$ canonical type I neuronal oscillators, called theta neurons [10]. The theta neuron model explicitly describes dynamic action potential generation and is equivalent to the normal form of a saddle node bifurcation on an invariant circle [11]. In contrast to previously considered network models that exhibit phase spaces of varying dimensionality [12], the phase space of our network model is a fixed $N$-torus. For these networks, we performed numerically exact calculations of the full spectrum of Lyapunov exponents, the rate of dynamical entropy production and the attractor dimension, and analyzed the statistics of the first Lyapunov vector. We found that both inhibitory networks and excitatory-inhibitory networks exhibit deterministic extensive chaos. The rate of dynamical entropy production in these networks was relatively high compared to experimentally measured information content of spike patterns in sensory cortices.

We studied large sparse networks of $N$ theta neurons arranged on directed Erdös-Rényi random graphs of fixed mean indegree $K$. The theta neuron model is a phase representation of the quadratic integrate and fire model [11]. Neurons are described by phases $\theta_{i} \in[-\pi, \pi]$, with spikes defined to occur when $\theta_{i}$ crosses $\pi$. The governing equation is

$$
\tau_{\mathrm{m}} \frac{\mathrm{d} \theta_{i}}{\mathrm{~d} t}=\left(1-\cos \theta_{i}\right)+I_{i}(t)\left(1+\cos \theta_{i}\right),
$$

with the membrane time constant $\tau_{\mathrm{m}}$ and the synaptic input current

$$
I_{i}(t)=\sqrt{K} I_{i}^{\text {ext }}+2 \tau_{\mathrm{m}} \sum_{j \in \operatorname{pre}(i)} \frac{J_{i j}}{\sqrt{K}} \delta\left(\theta_{j}(t)-\pi\right) .
$$

All neurons $i=1 \ldots N$ receive constant external excitatory currents $\sqrt{K} I_{i}^{\text {ext }}$. These were identical for all neurons in the excitatory (E) and inhibitory (I) population, respectively, and chosen to obtain a target average firing rate $\bar{\nu}_{E}=\bar{\nu}_{I}=\bar{\nu}$. At spike events of a presynaptic neuron $j \in$ pre $(i)$, neuron $i$ received non-delayed $\delta$-pulses of strength $J_{i j} / \sqrt{K}$, leading to step-like changes in the neuron's state. In inhibitory networks, every neuron was coupled to on average $K$ presynaptic neurons with $J_{I I}=-J_{0}$. In excitatory-inhibitory networks, the inhibitory neurons received inputs from on average $K$ inhibitory neurons with $J_{I I}=-\sqrt{1-\varepsilon^{2}} J_{0}$ and $K$ 
excitatory neurons with $J_{I E}=\varepsilon J_{0}$. The excitatory neurons received inputs from on average $K$ excitatory neurons with $J_{E E}=0.9 \varepsilon J_{0}$ and $K$ inhibitory neurons with $J_{E I}=$ $-\sqrt{1-(0.9 \varepsilon)^{2}} J_{0}$. At $\varepsilon=0$, all excitatory neurons are passive, since they only receive inputs from the inhibitory neurons without providing feedback. Increasing $\varepsilon$ activates the excitatory feedback loops, such that the magnitudes of input fluctuations $\sigma^{2}=J_{0}^{2} \bar{\nu}$ are preserved and identical to those in inhibitory networks [13].

From the analytical solutions of Eq. (1) with (2) we obtained an N-dimensional map of the neurons' phases between successive spike times in the network $\left\{t_{s}\right\}$ [13]. This map was used for event-based, numerically exact simulations of the network dynamics and yields the single spike Jacobian matrix $D\left(t_{s}\right)=\frac{\partial \vec{\theta}\left(t_{s}\right)}{\partial \vec{\theta}\left(t_{s-1}\right)}$ :

$$
D_{i j}\left(t_{s}\right)= \begin{cases}d_{i^{*}}\left(t_{s}\right) & \text { for } i=j=i^{*} \\ \sqrt{\frac{I_{i *}^{\text {ext }}}{I_{j^{*}}^{\text {ext }}}}\left(1-d_{i^{*}}\left(t_{s}\right)\right) & \text { for } i=i^{*}, j=j^{*} \\ \delta_{i j} & \text { otherwise, }\end{cases}
$$

where $j^{*}$ denotes the spiking neuron at time $t_{s}, i^{*} \in \operatorname{post}\left(j^{*}\right)$ the spike receiving neurons, $\delta_{i j}$ is the Kronecker symbol and

$$
d_{i^{*}}\left(t_{s}\right)=\frac{\left(\tan \left(\theta_{i^{*}}\left(t_{s}^{-}\right) / 2\right)\right)^{2}+\sqrt{K} I_{i^{*}}^{\text {ext }}}{\left(\tan \left(\theta_{i^{*}}\left(t_{s}^{-}\right) / 2\right)+J_{i^{*} j^{*}} / \sqrt{K}\right)^{2}+\sqrt{K} I_{i^{*}}^{\text {ext }}},
$$

where $\theta_{i^{*}}\left(t_{s}^{-}\right)$denotes the phase directly before spike reception [13].

The exact Jacobians (3) were used to numerically calculate all Lyapunov exponents in the standard Gram-Schmidt reorthogonalization procedure [13, 14]. From the Lyapunov exponents $\lambda_{1} \geq \ldots \geq \lambda_{N}$, we derived the KolmogorovSinai entropy production (Pesin identity): $H=\sum_{\lambda_{i}>0} \lambda_{i}$ and the attractor dimension (Kaplan-Yorke conjecture): $D=$ $d+S_{d} /\left|\lambda_{d+1}\right|$ (with $d$ the largest integer such that $S_{d}=$ $\left.\sum_{i=1}^{d} \lambda_{i} \geq 0\right)$ [15]. All calculations were repeated with 10 different initial conditions and network realizations. In all Figures below, standard errors are smaller than the symbol sizes.

The characteristics of inhibitory networks and excitatoryinhibitory networks in the balanced state are depicted in Fig. 11 Representative spike patterns and voltage traces $V(t)=\tan (\theta(t) / 2)$ illustrate the irregular, asynchronous firing and strong membrane potential fluctuations. In agreement with recent biological observations, the firing rate distributions were broad [16], indicating a substantial heterogeneity in the networks. Distributions of firing rates and coefficients of variation were identical in both types of networks.

We first characterized the dynamics of inhibitory networks in the balanced state (Fig. 2). These networks exhibited deterministic extensive chaos, characterized by finite positive maximal Lyapunov exponents and network size invariant Lyapunov spectra [13]. As a result, the attractor dimension and entropy production increased linearly with the number of neu-
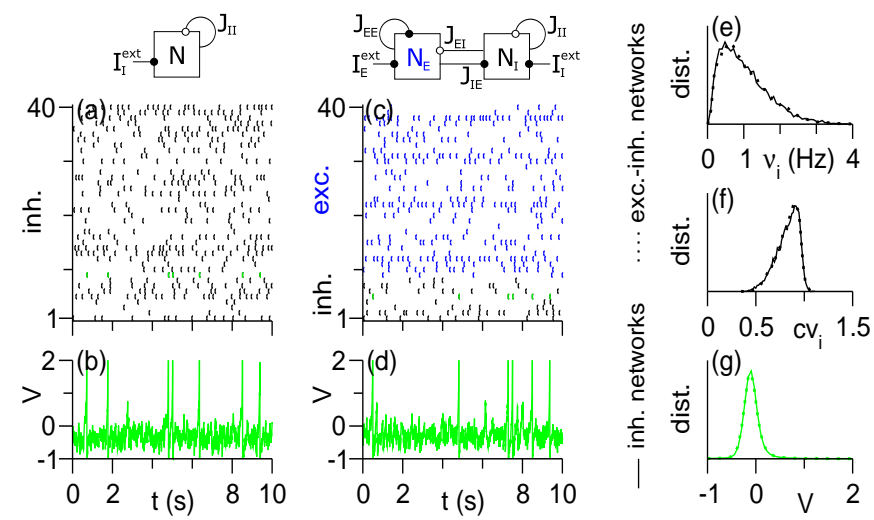

Figure 1: (color online) Characteristics of the balanced state in inhibitory and excitatory-inhibitory networks of theta neurons: (a,c) Spike patterns of 40 random neurons, $(b, d)$ Voltage traces of one random neuron, (e) Firing rate distributions, (f) Coefficient of variation distributions and (g) Stationary voltage distributions, (parameters: $N=10000, K=100, \bar{\nu}=1 \mathrm{~Hz}, J_{0}=1, \tau_{\mathrm{m}}=10 \mathrm{~ms}$, $r=0.9, \varepsilon=0.3, N_{E}=4 N_{I}$ )

rons in the networks. The range of the Lyapunov spectra increased with the average firing rate, such that the maximal Lyapunov exponent, entropy production and attractor dimension grew for larger firing rates. The chaotic attractors had a large dimensionality of the order of the phase space dimension $N$. The average entropy production (information loss) was of $\mathcal{O}(1)$ bit per spike per neuron. We remark that this is surprisingly high compared to the estimated information content of $\mathcal{O}(1)$ bit per spike in primary sensory cortices [17, 18].

Adding an excitatory population to the networks increased the intensity of balanced state chaos (Fig. 3), while the magnitude of input fluctuations and the basic firing statistics were preserved. When the excitatory neurons are passive $(\varepsilon=0)$, their dynamics is fully controlled by the inhibitory neurons. The positive part of the Lyapunov spectrum was then equivalent to that of an isolated inhibitory network of reduced size $N=N_{\text {I }}$ (dashed line). Hence, the maximal Lyapunov exponent was identical, whereas the entropy production per spike per neuron and the attractor dimension density were reduced accordingly by one fifth. Upon activation of the excitatory feedback loops $(\varepsilon>0)$ the entire Lyapunov spectrum changed. The maximal Lyapunov exponent, attractor dimension and entropy production increased with $\varepsilon$, demonstrating an intensification of the chaos.

Are all neurons involved equally and at all times in the chaotic dynamics despite their substantial firing heterogeneities? To answer this question, we studied the first Lyapunov vector $\overrightarrow{\delta \phi}(t)\left(\sum_{i=1}^{N} \delta \phi_{i}(t)^{2}=1\right)$. At each point in time, $\overrightarrow{\delta \phi}$ aligns with the direction in which initial perturbations exponentially grow with maximal asymptotic rate $\lambda_{\max }$. The Lyapunov vector was dominated by relatively small subsets of neurons that changed over time (Fig. 4). We analyzed the composition of these groups with two quantities: the participation ratio $P(t)=1 / \sum_{i=1}^{N} \delta \phi_{i}(t)^{4}$ quantify- 

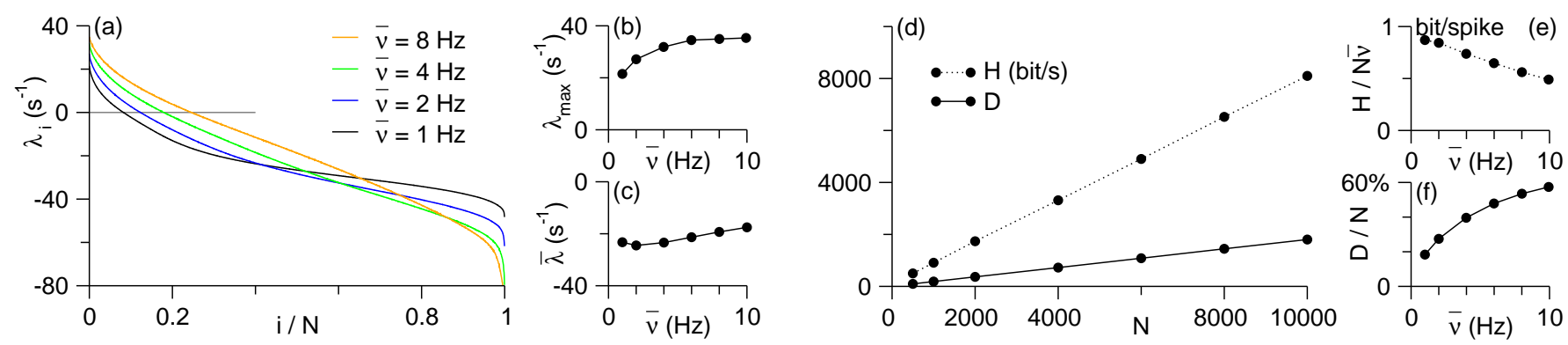

Figure 2: (color online) Extensive deterministic chaos in balanced inhibitory networks: (a) Full Lyapunov spectra for different average firing rates $\bar{\nu}$, collapsed for different network sizes, (b) Maximal Lyapunov exponent, (c) Mean Lyapunov exponent, (d) Entropy production rate $H$ and attractor dimension $D$ for various network sizes $N$, (e) Average entropy production per spike per neuron, (f) Attractor dimension density (parameters: $N=2000, K=100, \bar{\nu}=1 \mathrm{~Hz}, J_{0}=1, \tau_{\mathrm{m}}=10 \mathrm{~ms}$ )
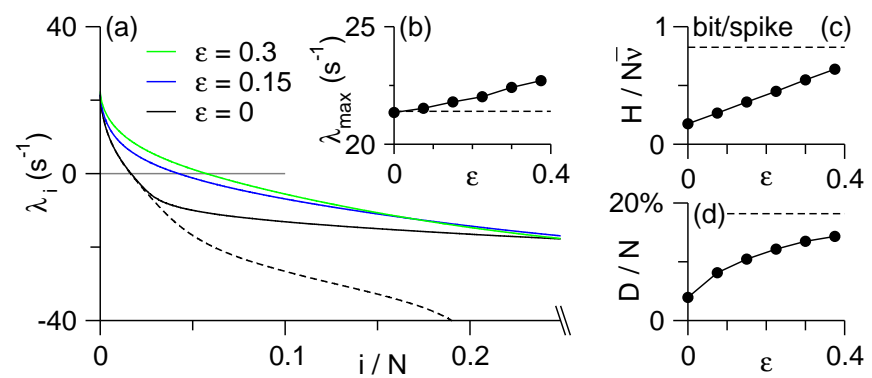

Figure 3: (color online) Chaotic dynamics in balanced excitatoryinhibitory networks at different excitatory feedback loop activations $\varepsilon$ : (a) Lyapunov spectra, (b) Maximal Lyapunov exponent, (c) Entropy production per spike per neuron, (d) Attractor dimension density (dashed lines: isolated inhibitory networks with $N=N_{I}$, parameters: $N_{\mathrm{I}}=2000, N_{\mathrm{E}}=8000, K=100, \bar{\nu}=1 \mathrm{~Hz}, J_{0}=1$, $\left.\tau_{\mathrm{m}}=10 \mathrm{~ms}\right)$
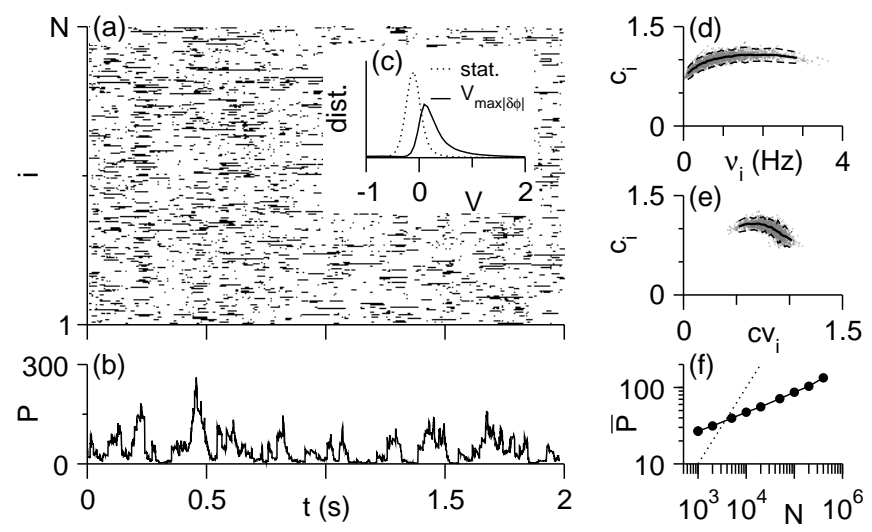

Figure 4: Temporal network chaos in balanced inhibitory networks: (a) First Lyapunov vector $\overrightarrow{\delta \phi}(t)$ (marked black if $\left|\delta \phi_{i}(t)\right|>1 / \sqrt{N}$ ), (b) Participation ratio $P(\mathrm{t})$ of first Lyapunov vector, (c) Voltage distribution of neurons with largest Lyapunov vector elements (dotted line: stationary distribution) (d,e) Neurons' chaos indices $c_{i}$ versus firing rates $\nu_{i}$ and coefficients of variation $c v_{i}$ (straight line: mean, dashed lines: $\pm 2 \mathrm{std}$ ), (f) Average participation ratio $\bar{P}$ vs. network size $N$ (dotted line: guide to the eye for $\bar{P} \sim N$ ), (parameters: $\left.N=2000, K=100, \bar{\nu}=1 \mathrm{~Hz}, J_{0}=1, \tau_{\mathrm{m}}=10 \mathrm{~ms}\right)$ ing the size of a group at time $t$ [19], and the chaos index $c_{i}=\sqrt{N\left\langle\delta \phi_{i}(t)^{2}\right\rangle_{t}}$ quantifying the time-averaged participation of individual neurons. The participation ratio exhibited substantial fluctuations indicating strongly varying group sizes. For the considered rates $1 \mathrm{~Hz} \leq \bar{\nu} \leq 10 \mathrm{~Hz}$, the timeaveraged participation ratio $\bar{P}=\langle P(t)\rangle_{t}$ obeyed a sublinear scaling $\bar{P} \sim N^{\alpha}$, with $0.25 \leq \alpha \leq 0.5$ [13]. Thus, although the average group size grew with network size, the fraction of most unstable neurons decreased algebraically as $N^{\alpha-1}$. The chaos indices were essentially independent of the neurons' firing rates and $\mathrm{cv}$ values. This means that statistical firing irregularity is unrelated to chaoticity. Thus, although at any time only a small group of neurons constitutes the most unstable direction, over time all neurons participated almost equally in the chaotic dynamics.

To understand when the neurons were the most sensitive to state perturbations, we examined the voltage distribution of neurons when exhibiting the largest Lyapunov vector element. This distribution compared to the stationary distribution was shifted towards the spike generating instability, marking it as an important source of instability of the collective dynamics (Fig. 4(c)).

The chaotic dynamics of balanced networks described here shares many features with classical examples of spatiotemporal chaos [19]. Balanced networks exhibit a sizeinvariant Lyapunov spectrum and extensive entropy production rate and attractor dimension. The chaotic degrees of freedom are confined to small and varying groups of elements. The type of this localization, however, differs from classical examples of spatio-temporal chaos. While classically, the participation ratio is constant for large $N$, we here found an algebraic sublinear dependence. This behavior apparently reflects the non-local graph structure of the systems and might thus be termed temporal network chaos. In fact, chaos in balanced networks is induced by the complex network connectivity: neither the isolated units nor a fully connected network of theta neurons exhibit chaotic dynamics. Extensive chaos is expected in spatially extended systems, that can be decomposed into weakly interacting subsystems, whose number grows linearly with system size [20]. As this is not fulfilled for network 
dynamical systems on random graphs, extensive chaos in our networks is not a trivial property. Globally coupled networks for instance can in fact exhibit non-extensive chaos [21]. The extensivity found for balanced chaos likely reflects the lack of correlation between neurons in this state. Even in densely connected random networks, pairwise correlations are expected to vanish in the large system limit [22]. Then, the invariant measure will factorize and the attractor becomes $\mathcal{O}(N)$ dimensional in the large system limit. It should be noted that the studied networks may be viewed as subunits of cortical networks that can be approximated with random connectivity. In spatially extended networks, composed of such subunits, extensivity may be expected for standard reasons.

Our results reveal that the dynamics of balanced networks strongly depends on the single neuron dynamics. While we found that theta neurons in the balanced state exhibit conventional extensive chaos, previous studies of networks of leaky integrate and fire neurons demonstrated stable chaos [8]. Opposed to the leaky integrate and fire model, the theta neuron model incorporates the dynamic instability underlying spike initiation. The membrane potential distribution of the neurons when most sensitive to perturbations is indeed shifted towards this instability. Our results thus indicate, that this feature of single neuron dynamics renders the collective dynamics of balanced networks robustly chaotic.

The strikingly large entropy production we found raises questions about the neural code in cortical networks. If the entropy production is of the same order of magnitude as sensory information carried by spikes, sensory information would be hard to maintain in spike patterns beyond the immediate stimulus response. In fact, a study of spike timing in barrel cortex showed that information about whisker deflections is encoded to $83 \%$ in the first spike after the stimulus and reduced in each successive spike by roughly two thirds [18]. Since the theta neuron model is the canonical form of type I excitability, our results are expected to be representative of a wide range of single neuron dynamics. The reliable generation of long and precisely timed spike sequences by cortical networks would then be unlikely.

The fat chaotic attractors in balanced networks described here might serve as a rich repertoire of complex states the network is rapidly plowing through. Our study lays the foundation for future research on dynamic stabilization mechanisms for unstable states or reliable computations on short time scales on existing stable directions.

We thank E. Bodenschatz, P. Cvitanović, T. Geisel, M. Gutnick, H. Sompolinsky, M. Timme and C. van Vreeswijk for fruitful discussions. M. M. acknowledges the hospitality of J. Golowasch and F. Nadim at Rutgers University. This work was supported by BMBF (01GQ07113) and GIF (90617.1/2006).
Note added in proof: Our findings of a strongly chaotic dynamics is consistent with novel experimental evidence of a high sensitivity to perturbations in cortical networks in vivo [23].

* Electronic address: monte@nld.ds.mpg.de

[1] B. D. Burns and A. C. Webb, Proc. R. Soc. B 194, 211 (1976); W. R. Softky and C. Koch, J. Neurosci. 13, 334 (1993)

[2] Z. F. Mainen and T. J. Sejnowski, Science 268, 1503 (1995)

[3] G. R. Holt et al., J. Neurophysiol. 75, 1806 (1996)

[4] M. N. Shadlen and W. T. Newsome, Curr. Opin. Neurobiol. 4, 569 (1994); M. N. Shadlen and W. T. Newsome, J. Neurosci. 18, 3870 (1998); W. R. Softky, Curr. Opin. Neurobiol. 5, 239 (1995)

[5] C. van Vreeswijk and H. Sompolinsky, Science 274, 1724 (1996); C. van Vreeswijk and H. Sompolinsky, Neural Comput. 10, 1321 (1998)

[6] Y. Shu, A. Hasenstaub and D. A. McCormick, Nature 423, 288 (2003); B. Haider et al., J. Neurosci. 26, 4535 (2006); R. W. Berg, A. Alaburda and J. Hounsgaard, Science 315, 390 (2007)

[7] M. Tsodyks and T. Sejnowski, Network 6, 111 (1995); D. J. Amit and N. Brunel, Cereb. Cortex 7, 237 (1997); D. J. Amit and N. Brunel, Network 8, 373 (1997); T. W. Troyer and K. D. Miller, Neural Comput. 9, 971 (1997); N. Brunel, J. Comput. Neurosci. 8183 (2000); A. Lerchner et al., Neural Comput. 18, 634 (2006)

[8] S. Jahnke, R. M. Memmesheimer and M. Timme, Phys. Rev. Lett. 100, 048102 (2008); R. Zillmer, N. Brunel and D. Hansel, Phys. Rev. E 79, 031909 (2009)

[9] A. Politi et al., Europhys. Lett. 22, 571 (1993)

[10] P. E. Latham et al., J. Neurophysiol. 83, 808 (2000); R. Osan and B. Ermentrout, Neurocomputing 38, 789 (2001); D. Hansel and G. Mato, Neural Comput. 15, 1 (2003); B. S. Gutkin, J. Jost and H. C. Tuckwell, Europhys. Lett. 81, 20005 (2008)

[11] G. B. Ermentrout and N. Kopell, SIAM J. Appl. Math. 46, 233 (1986); B. S. Gutkin and G. B. Ermentrout, Neural Comput. 10, 1047 (1998)

[12] P. Ashwin and M. Timme, Nonlinearity 18, 2035 (2005)

[13] See EPAPS Document No. xxx.yyy for more details.

[14] G. Benettin et al., Meccanica 15, 21 (1980)

[15] J.-P. Eckmann and D. Ruelle, Rev. Mod. Phys. 57, 617 (1985)

[16] T. Hromadka, M. R. DeWeese and A. M. Zador, PLOS Biol. 6, 0124 (2008)

[17] A. Borst and F. E. Theunissen, Nat. Neurosci. 2, 947 (1999)

[18] S. Panzeri et al., Neuron 29, 769 (2001)

[19] M. C. Cross and P. C. Hohenberg, Rev. Mod. Phys. 65, 851 (1993)

[20] D. Ruelle, Commun. Math. Phys. 87, 287 (1982)

[21] K. A. Takeuchi, F. Ginelli and H. Chate, Phys. Rev. Lett. 103, 154103 (2009)

[22] B. Derrida, E. Gardner and A. Zippelius, Europhys. Lett. 4, 167 (1987); J. Herz, Neural Comput. 22, 427 (2010); A. Renart et al., Science 327, 587 (2010)

[23] M. London et al., Nature 466, 123 (2010) 